\title{
Study of deuteron-proton backward elastic scattering at intermediate energies.
}

\author{
Ladygina N. ${ }^{1 \star}$ \\ 1 Joint Institute for Nuclear Research, Dubna, Russia \\ * nladygina@jinr.ru
}

October 1, 2019

盟战

Proceedings for the 24th edition of European Few Body Conference, Surrey, UK, 2-4 September 2019

\begin{abstract}
We study deuteron- proton elastic scattering in the deuteron energy range between 500 $\mathrm{MeV}$ and $2 \mathrm{GeV}$ at the cms scattering angle $\theta^{*} \geq 140^{\circ}$. The reaction is considered in the relativistic multiple scattering expansion framework. The four reaction mechanisms are included into consideration: one-nucleon exchange, single scattering, double scattering, and the term corresponding to the delta excitation in the intermediate state.

The model is applied to describe the angular dependence of the differential cross section at the deuteron energies of between 880 and $1300 \mathrm{MeV}$. Also the energy dependence of the differential cross section and polarisation observables such as tensor analyzing power $T_{20}$ and polarization transfer from the deuteron to proton $\chi$ are considered at the scattering angle equal to $180^{\circ}$. Contributions of the different reaction mechanisms into the reaction amplitude are demonstrated in comparison with the existing experimental data.
\end{abstract}

\section{Contents}

1 Introduction 1

2 General formalism 2

$\begin{array}{lll}3 & \text { Results } & 7\end{array}$

4 Conclusion 9

References 9

\section{Introduction}

Elastic deuteron-proton scattering is the simplest example of the hadron nucleus collision. Nowadays, a significant amount of the experimental data has been accumulated in a wide 
energy range both with unpolarized and polarized beams. However, we do not have any theory to describe the data for the energies above a few hundred $\mathrm{MeV}$, especially, at backward scattering angles.

A good theoretical description of the deuteron-nucleon process was obtained at low energies, where the multiple scattering formalism based on the solution of the Faddeev equations, has been applied to this problem [1]. However, at the nucleon energies above $130 \mathrm{MeV}$ there is some discrepancy between the experimental data and theoretical predictions in the minimum of the differential cross section [2].

The Glauber theory taking into account both single and double nucleon-nucleon interaction successfully describes the differential cross sections of the dp-elastic scattering at small angles [3]- [4]. But it does not properly work at larger scattering angles.

In 1969 A.Kerman and L.Kisslinger supposed that resonances can play an important role in deuteron-proton backward elastic scattering [5]. Later the double-scattering diagram with $\Delta$-isobar in the intermediate state was taken into account in dp- backward scattering. The significant contribution of this term to the reaction amplitude was demonstrated in refs. [6][8]. However, the double scattering with nucleon in an intermediate state was not considered in these papers. Perhaps, it was the reason why the description of the differential cross sections energy dependence was not good enough.

The effort to take the $\Delta$-isobar into account in order to describe dp-elastic scattering was also done in [9], [10]. In these papers deuteron-proton scattering was considered in a whole angular range, not only at $\theta^{*}=180^{\circ}$. Unfortunately, the process was studied at low energies, $T_{d}<200 \mathrm{MeV}$, where the $\Delta$-isobar excitation effects are negligible.

We have previously proposed to use a model based on the multiple expansion of the reaction amplitude in powers of the nucleon-nucleon t-matrix [11]- [13]. Here we apply the model for description of the deuteron-proton elastic scattering in backward kinematics.

\section{General formalism}

According to the three-body collision theory, the amplitude of the deuteron-proton elastic scattering $\mathcal{J}$ is defined by the matrix element of the transition operator $U_{11}$ :

$$
\begin{aligned}
U_{d p \rightarrow d p}= & \delta\left(E_{d}+E_{p}-E_{d}^{\prime}-E_{p}^{\prime}\right) \mathcal{J}= \\
& <1(23)\left|\left[1-P_{12}-P_{13}\right] U_{11}\right| 1(23)>.
\end{aligned}
$$

Here, the state $\mid 1(23)>$ corresponds to the configuration, when nucleons 2 and 3 form the deuteron state and nucleon 1 is free. The permutation operators for two nucleons $P_{i j}$ reflects the fact that the initial and final states are antisymmetric due to the two particles exchange.

The transition operators for rearrangement scattering are defined by the Alt-GrassbergerSandhas equations:

$$
\begin{array}{llr}
U_{11} & = & t_{2} g_{0} U_{21}+t_{3} g_{0} U_{31}, \\
U_{21}= & g_{0}^{-1}+t_{1} g_{0} U_{11}+t_{3} g_{0} U_{31}, \\
U_{31}= & g_{0}^{-1}+t_{1} g_{0} U_{11}+t_{2} g_{0} U_{21}
\end{array}
$$

where $t_{1}=t(2,3)$, etc., is the $t$-matrix of the two-nucleon interaction and $g_{0}$ is the free threeparticle propagator. The indices $i j$ for the transition operators $U_{i j}$ denote free particles $i$ and $j$ in the final and initial states, respectively.

Iterating these equations up to the $t_{i}$-second-order terms, we can present the reaction amplitude as a sum of the four contributions:

$$
\mathcal{J}_{d p \rightarrow d p}=\mathcal{J}_{\mathrm{ONE}}+\mathcal{J}_{\mathrm{SS}}+\mathcal{J}_{\mathrm{DS}}+\mathcal{J}_{\Delta}
$$




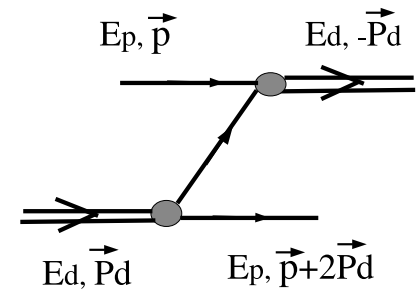

(a)

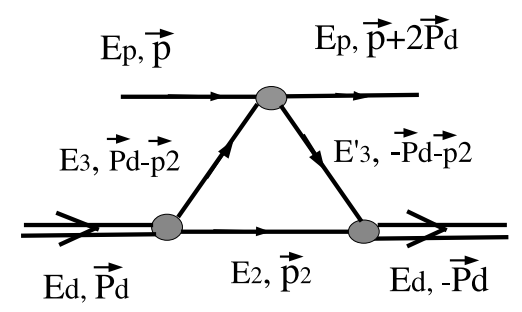

(b)

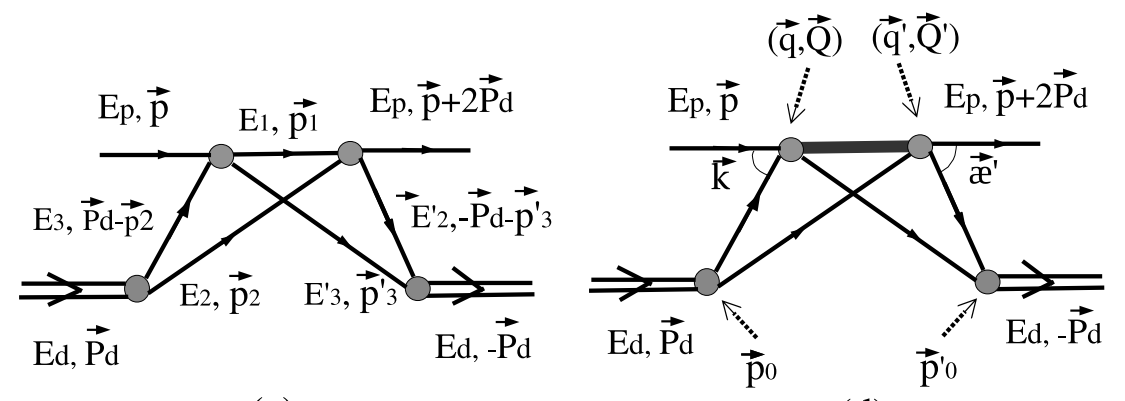

(c)

(d)

Figure 1: The diagrams included into consideration: (a) the one-nucleon exchange diagram; (b) the single scattering diagram; (c) the double scattering diagram with a nucleon in the intermediate state; (d) the double scattering diagram with $\Delta$-isobar in the intermediate state.

one-nucleon exchange, single scattering, double scattering, and rescattering with $\Delta$-excitation in the intermediate state.

The first term in the $d p$-elastic scattering amplitude $\mathcal{J}$ in Eq.(3) is the one nucleon exchange (ONE) term.

$$
\mathcal{J}_{\text {ONE }}=-2<1(23)\left|P_{12} g_{0}^{-1}\right| 1(23)>
$$

The corresponding diagram is presented in Fig.1a. Applying the definitions of the wave function of a moving deuteron and three- nucleon free propagator, we can write ONE amplitude in the following form:

$$
\begin{aligned}
\mathcal{J}_{O N E}= & -\frac{1}{2}\left(E_{d}-E_{p}-\sqrt{m_{N}^{2}+\vec{p}^{2}-\vec{P}_{d}^{2}}\right) . \\
& <\vec{p}^{\prime} m^{\prime} ;-\vec{P}_{d} \mathcal{M}_{d}^{\prime}\left|\Omega_{d}^{\dagger}(23)\left[1+\left(\sigma_{1} \sigma_{2}\right)\right] \Omega_{d}(23)\right| \vec{P}_{d} \mathcal{M}_{d} ; \vec{p} m>,
\end{aligned}
$$

where the definition of the permutation operator in spin space $P_{12}(\sigma)=\frac{1}{2}\left[1+\left(\sigma_{1} \sigma_{2}\right)\right]$ has been applied.

All the calculations are performed in the deuteron Breit frame, where the deuterons move in opposite directions with equal momenta (Fig.1). It allows us to minimize the relative momenta of the nucleons in the both deuterons. As a consequence, the non-relativistic deuteron wave function can be applied in the energy range under consideration.

In the rest frame the non-relativistic wave function of the deuteron depends only on one variable $\vec{p}_{0}$, which is the relative momentum of the outgoing proton and neutron:

$$
<\mu_{p} \mu_{n}\left|\Omega_{d}\right| \mathcal{M}_{d}>=\frac{1}{\sqrt{4 \pi}}<\mu_{p} \mu_{n}\left|\left\{u\left(p_{0}\right)+\frac{w\left(p_{0}\right)}{\sqrt{8}}\left[3\left(\sigma_{1} \hat{p}_{0}\right)\left(\sigma_{2} \hat{p}_{0}\right)-\left(\sigma_{1} \sigma_{2}\right)\right]\right\}\right| \mathcal{M}_{d}>
$$


where $u\left(p_{0}\right)$ and $w\left(p_{0}\right)$ describe the $S$ and $D$ components of the deuteron wave function [14], [15], [16], $\hat{p}_{0}$ is the unit vector in $\vec{p}_{0}$ direction.

In order to get the wave function of the moving deuteron, it is necessary to apply the Lorenz transformations for the kinematical variables and Wigner rotations for the spin states. This procedure has been expounded in ref. [11]. The proton-neutron relative momenta for the initial $\vec{p}_{0}$ and final $\vec{p}_{0}^{\prime}$ deuterons are expressed as:

$$
\vec{p}_{0}=\vec{p}+\vec{P}_{d}\left[1+\frac{E_{n}+E^{*}}{E_{p}+E_{n}+E^{*}}\right], \quad \vec{p}_{0}^{\prime}=\vec{p}+\vec{P}_{d}\left[1-\frac{E_{n}+E^{*}}{E_{p}+E_{n}+E^{*}}\right] .
$$

Here $E_{n}=\sqrt{m_{N}^{2}+\vec{p}^{2}-\vec{P}_{d}^{2}}$ and $E^{*}=\sqrt{\left(E_{p}+E_{n}\right)^{2}-\vec{P}_{d}^{2}} / 2$ are the struck neutron energy in the moving deuteron frame and rest deuteron frame, respectively. Note, that $\left|\vec{p}_{0}\right|=\left|\vec{p}_{0}^{\prime}\right|$.

The next term in the $d p$-elastic scattering amplitude Eq.(3) is the single scattering one.

$$
\mathcal{J}_{S S}=2<1(23)\left|\left[1-P_{12}\right] t_{3}\right| 1(23)>
$$

The corresponding diagram is presented in Fig.(1b). Following the standard procedure we get the expression for the single scattering amplitude:

$$
\begin{aligned}
& \mathcal{J}_{S S}=\int d \vec{q}^{\prime}<-\vec{P}_{d} \mathcal{M}_{d}^{\prime}\left|\Omega_{d}^{\dagger}\right| \vec{q}^{\prime} m^{\prime \prime},-\vec{P}_{d}-\vec{q}^{\prime} m_{3}^{\prime}> \\
&<\vec{p}^{\prime} m^{\prime},-\vec{P}_{d}-\vec{q}^{\prime}\left|\frac{3}{2} t_{12}^{1}+\frac{1}{2} t_{12}^{0}\right| \vec{p} m, \vec{P}_{d}-\vec{q}^{\prime} m_{2}^{\prime}><\vec{q}^{\prime} m^{\prime \prime}, \vec{P}_{d}-\vec{q}^{\prime} m_{2}^{\prime}\left|\Omega_{d}\right| \vec{P}_{d} \mathcal{M}_{d}>.
\end{aligned}
$$

The relative momenta of two nucleons for the initial and final deuterons are

$$
\vec{p}_{0}=\vec{q}^{\prime}-\vec{P}_{d} \frac{E_{2}+E^{*}}{E_{2}+E_{3}+2 E^{*}} \quad \vec{p}_{0}^{\prime}=\vec{q}^{\prime}+\vec{P}_{d} \frac{E_{2}+E^{*}}{E_{2}+E_{3}^{\prime}+2 E^{\prime *}},
$$

where the nucleons energies $E_{2}, E_{3}, E_{3}^{\prime}$ in the reference frame are defined by the standard manner (Fig.1b)

$$
E_{2}=\sqrt{m_{N}^{2}+\vec{q}^{\prime 2}}, \quad E_{3}=\sqrt{m_{N}^{2}+\left(\vec{P}_{d}-\vec{q}^{\prime}\right)^{2}}, \quad E_{3}^{\prime}=\sqrt{m_{N}^{2}+\left(\vec{P}_{d}+\vec{q}^{\prime}\right)^{2}}
$$

and these energies in the center-of-mass of the two nucleons forming the initial and final deuterons are equal, correspondingly, to

$$
E^{*}=\frac{1}{2} \sqrt{\left(E_{2}+E_{3}\right)^{2}-\vec{P}_{d}^{2}}, \quad E^{* *}=\frac{1}{2} \sqrt{\left(E_{2}+E_{3}^{\prime}\right)^{2}-\vec{P}_{d}^{2}} .
$$

The nucleon-nucleon scattering is described by the t-matrix $t_{i j}^{T}$. We use the parameterization of this matrix offered by Love and Franey [17]. This is the on-shell NN t-matrix defined in the center-of-mass:

$$
\begin{aligned}
& <\boldsymbol{x}^{* \prime} \mu_{1}^{\prime} \mu_{2}^{\prime}\left|t_{c . m .}\right| \boldsymbol{x}^{*} \mu_{1} \mu_{2}>=<\boldsymbol{x}^{* \prime} \mu_{1}^{\prime} \mu_{2}^{\prime} \mid A+B\left(\sigma_{1} \hat{N}^{*}\right)\left(\sigma_{2} \hat{N}^{*}\right)+ \\
& C\left(\sigma_{1}+\sigma_{2}\right) \cdot \hat{N}^{*}+D\left(\sigma_{1} \hat{q}^{*}\right)\left(\sigma_{2} \hat{q}^{*}\right)+F\left(\sigma_{1} \hat{Q}^{*}\right)\left(\sigma_{2} \hat{Q}^{*}\right) \mid \boldsymbol{x}^{*} \mu_{1} \mu_{2}>.
\end{aligned}
$$

The orthonormal basis $\left\{\hat{q}^{*}, \hat{Q}^{*}, \hat{N}^{*}\right\}$ is a combination of the nucleon relative momenta in the initial $\boldsymbol{x}^{*}$ and final $\boldsymbol{x}^{*}$ states:

$$
\hat{q}^{*}=\frac{x^{*}-x^{* \prime}}{\left|x^{*}-x^{* \prime}\right|}, \quad \hat{Q}^{*}=\frac{x^{*}+x^{* \prime}}{\left|x^{*}+x^{* \prime}\right|}, \quad \hat{N}^{*}=\frac{x^{*} \times x^{* \prime}}{\left|x^{*} \times x^{* \prime}\right|} .
$$

The amplitudes $A, B, C, D, F$ are the functions of the center-of-mass energy and scattering angle. The radial parts of these amplitudes are taken as a sum of Yukawa terms. A new 
fit of the model parameters [18] was done in accordance with the phase-shift-analysis data SP07 [19].

Since the matrix elements are expressed via the effective $N N$-interaction operators sandwiched between the initial and final plane-wave states, this construction can be extended to the off-shell case allowing the initial and final states to get the current values of $\boldsymbol{x}$ and $\boldsymbol{x}^{\prime}$. Obviously, this extrapolation does not change the general spin structure.

The double scattering contribution (Fig.1c) is defined by a deuteron wave function and two nucleon-nucleon t-matrixes. Also we have here three-nucleon propagator:

$$
\begin{aligned}
& \mathcal{J}_{D S}=\int d \overrightarrow{p_{2}} d \overrightarrow{p_{3}^{\prime}}<-\vec{P}_{d} \mathcal{M}_{d}^{\prime}\left|\Omega_{d}^{\dagger}\right|-\vec{P}_{d}-\vec{p}_{3}^{\prime} m_{2}^{\prime}, \vec{p}_{3}^{\prime} m_{3}^{\prime}> \\
& <\vec{p}^{\prime} m^{\prime},-\vec{P}_{d}-\vec{p}_{3}^{\prime} m_{2}^{\prime}, \vec{p}_{3}^{\prime} m_{3}^{\prime} \mid \\
& \frac{t_{3(N N)}^{1}\left(E^{\prime}\right) t_{2(N N)}^{1}(E)+\left[t_{3(N N)}^{1}\left(E^{\prime}\right)+t_{3(N N)}^{0}\left(E^{\prime}\right)\right]\left[t_{2(N N)}^{1}(E)+t_{2(N N)}^{0}(E)\right] / 4}{E_{d}+E_{p}-E_{1}-E_{2}-E_{3}^{\prime}+i \varepsilon} \\
& \left|\vec{p} m, \vec{p}_{2} m_{2}, \vec{P}_{d}-\vec{p}_{2} m_{3}><\vec{p}_{2} m_{2}, \vec{P}_{d}-\vec{p}_{2} m_{3}\right| \Omega_{d} \mid \vec{P}_{d} \mathcal{M}_{d}>.
\end{aligned}
$$

The argument of the $N N$-matrix is defined as the three-nucleon on-shell energy excluding the energy of the nucleon which does not participate in the interaction:

$$
E=E_{d}+E_{p}-E_{2}, \quad E^{\prime}=E_{d}+E_{p}-E_{3}^{\prime} .
$$

The structure of the delta amplitude (Fig.1d) looks like the double-scattering one. But here we have $N N \rightarrow \Delta N$ matrixes instead the nucleon-nucleon matrixes and $N N \Delta$-propagator instead three-nucleon one.

$$
\begin{aligned}
& \mathcal{J}_{\Delta}=2 \int d \overrightarrow{p_{2}} d \overrightarrow{p_{3}^{\prime}} d E_{\Delta} d \overrightarrow{p_{\Delta}} \delta\left(E_{\Delta}-\sqrt{\mu^{2}+\overrightarrow{p_{\Delta}^{2}}}\right) \delta\left(\vec{p}+\overrightarrow{P_{d}}-\overrightarrow{p_{2}}-\overrightarrow{p_{3}}-\overrightarrow{p_{\Delta}}\right)_{1}<\frac{1}{2} \tau^{\prime} \frac{1}{2} m^{\prime} \vec{p}^{\prime} \mid \\
& { }_{23}<00 ;-\vec{P}_{d} 1 \mathcal{M}_{d}^{\prime}\left|\Omega_{d}^{\dagger}\left[1-P_{12}\right]\right| t_{3(N \Delta)}\left(E^{\prime}\right) \frac{1}{E-E_{2}-E_{3}^{\prime}-E_{\Delta}+i \Gamma\left(E_{\Delta} / 2\right)} \mid \Psi_{\vec{p}_{\Delta}}\left(E_{\Delta}\right)>_{1} \\
& \left|\frac{1}{2} \tau_{2} \frac{1}{2} m_{2} \vec{p}_{2} ; \frac{1}{2} \tau_{3} \frac{1}{2} m_{3} \vec{p}_{3}>_{23} 23<\frac{1}{2} \tau_{2} \frac{1}{2} m_{2} \vec{p}_{2} ; \frac{1}{2} \tau_{3} \frac{1}{2} m_{3} \vec{p}_{3}\right| \\
& { }_{1}<\Psi_{\vec{p}_{\Delta}}\left(E_{\Delta}\right)|| t_{2(N \Delta)}(E)\left[1-P_{13}\right] \Omega_{d}\left|\overrightarrow{P_{d}} 1 \mathcal{M}_{d} ; 00>_{23}\right| \frac{1}{2} \tau \frac{1}{2} m \vec{p}>_{1}
\end{aligned}
$$

Here a full set of the particles quantum numbers was included into the amplitude definition. Isospin and spin quantum numbers are marked by $\tau$ and $m$ or $M$, respectively. The indexes near the bracket correspond to the particles numbers.

The distribution function of the delta energy

$$
\left|\Psi_{\vec{p}_{\Delta}}\left(E_{\Delta}\right)><\Psi_{\vec{p}_{\Delta}}\left(E_{\Delta}\right)\right|=\rho\left(E_{\Delta}\right)
$$

is defined through the delta width $\Gamma(\mu)$ :

$$
\rho(\mu)=\frac{1}{2 \pi} \frac{\Gamma(\mu)}{\left(E_{\Delta}(\mu)-E_{\Delta}\left(m_{\Delta}\right)\right)^{2}+\Gamma^{2}(\mu) / 4},
$$

where $\mu^{2}=E_{\Delta}^{2}-\vec{p}_{\Delta}^{2}$ is the squared four-momentum of the delta. The delta width is energy dependent. We use, here, the standart parameterization of $\Gamma(\mu)$ taking into account the $\Delta$ off-shell corrections:

$$
\Gamma(\mu)=\Gamma_{0} \frac{p^{3}\left(\mu^{2}, m_{\pi}^{2}\right)}{p^{3}\left(m_{\Delta}^{2}, m_{\pi}^{2}\right)} \cdot \frac{p^{2}\left(m_{\Delta}^{2}, m_{\pi}^{2}\right)+\gamma^{2}}{p^{2}\left(\mu^{2}, m_{\pi}^{2}\right)+\gamma^{2}} .
$$




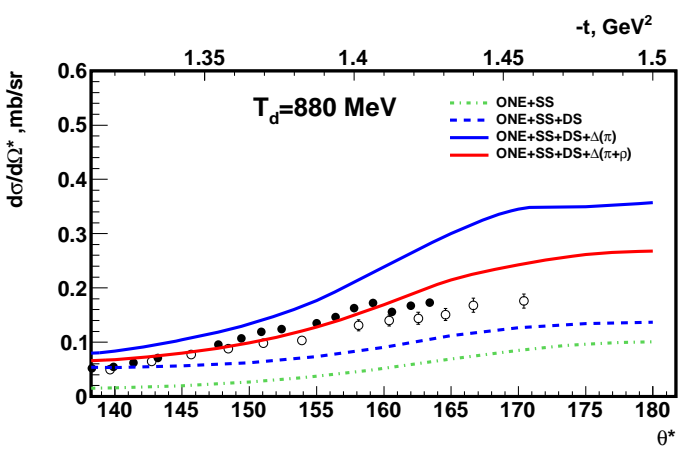

Figure 2: The angular dependence of the differential cross section at the deuteron energy $T_{d}=880 \mathrm{MeV}$. The data are from $\circ-[20]$ at $T_{d}=850 \mathrm{MeV} \bullet-[21]$ at $T_{d}=940 \mathrm{MeV}$

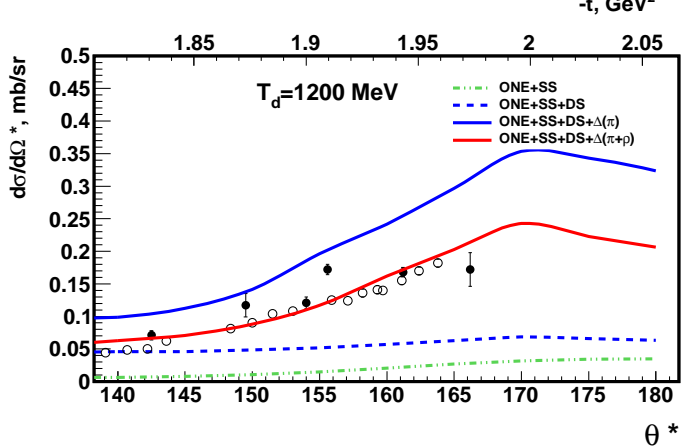

Figure 4: The angular dependence of the differential cross section at the deuteron energy at $T_{d}=1200$ $\mathrm{MeV}$. The data are from $\circ-[20]$ at $T_{d}=1169 \mathrm{MeV}$, - - [23] at $T_{d}=1200 \mathrm{MeV}$.

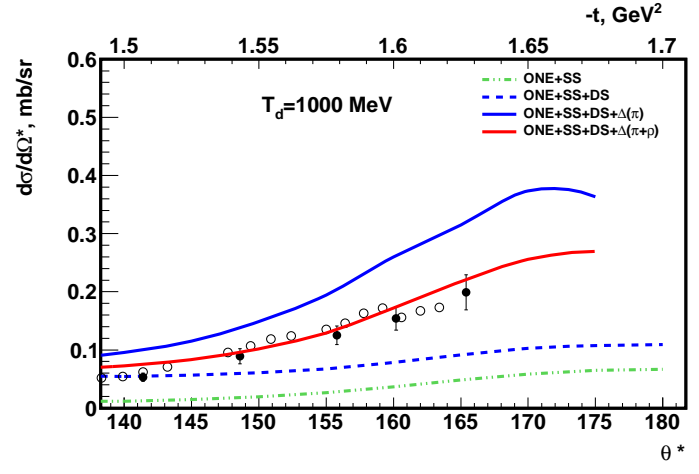

Figure 3: The angular dependence of the differential cross section at the deuteron energy at $T_{d}=1000$ $\mathrm{MeV}$. The data are from o- [20] at $T_{d}=940 \mathrm{MeV}$, - [22] at $T_{d}=1169 \mathrm{MeV}$.

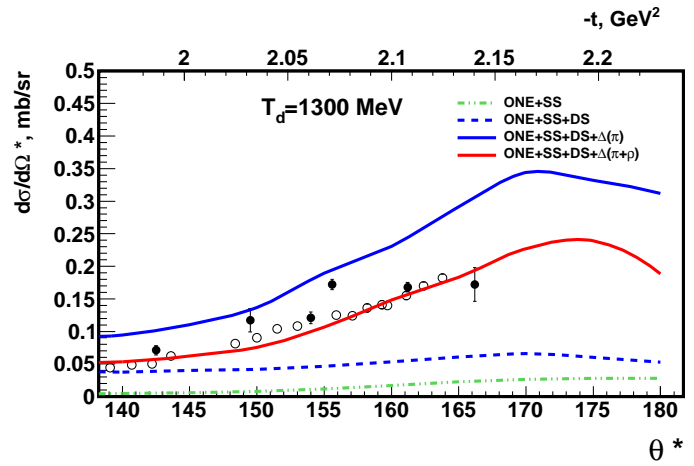

Figure 5: The angular dependence of the differential cross section at the deuteron energy $T_{d}=1300 \mathrm{MeV}$. The data are from $\circ-[20]$ at $T_{d}=1169 \mathrm{MeV}, \bullet-[23]$ at $T_{d}=1200 \mathrm{MeV}$.

where $p\left(x^{2}, m_{\pi}^{2}\right)$ is the momentum in the $\pi N$-centere-of-mass:

$$
p\left(x^{2}, m_{\pi}^{2}\right)=\sqrt{\left(x^{2}+m_{N}^{2}-m_{\pi}^{2}\right)^{2} / 4 x^{2}-m_{N}^{2}} .
$$

In our calculation we use the following value:

$$
\Gamma_{0}=0.120 \mathrm{GeV}, \quad \gamma=0.200 \mathrm{GeV}, \quad m_{\Delta}=1.232
$$

In the Born approximation the $N N \rightarrow N \Delta$ t-matrix can be replaced with the corresponding potential:

$$
<\vec{p}, \frac{1}{2} m, \frac{1}{2} \tau\left|t_{(N \Delta)}(E)\right| \Psi_{\vec{p}_{\Delta}}\left(E_{\Delta}\right)>\approx<\vec{p}, \frac{1}{2} m, \frac{1}{2} \tau\left|V_{(N \Delta)}(E)\right| \Psi_{\vec{p}_{\Delta}}\left(E_{\Delta}\right)>
$$

The potential for the $N N \rightarrow N \Delta$ transition is based on the $\pi$ - and $\rho$ - exchanges:

$$
\begin{aligned}
V_{\beta \alpha}^{(\pi)} & =-\frac{f_{\pi} f_{\pi}^{*}}{m_{\pi}^{2}} F_{\pi}^{2}(t) \frac{q^{2}}{m_{\pi}^{2}-t}(\vec{\sigma} \cdot \hat{q})(\vec{S} \cdot \hat{q})(\vec{\tau} \cdot \vec{T}) \\
V_{\beta \alpha}^{(\rho)} & =-\frac{f_{\rho} f_{\rho}^{*}}{m_{\rho}^{2}} F_{\rho}^{2}(t) \frac{q^{2}}{m_{\rho}^{2}-t}\{(\vec{\sigma} \vec{S})-(\vec{\sigma} \cdot \hat{q})(\vec{S} \cdot \hat{q})\}(\vec{\tau} \cdot \vec{T})
\end{aligned}
$$

Here, $t$ is the four transfer momentum and $\vec{q}$ is the corresponding three transfer momentum. The operators $\vec{\sigma}(\vec{\tau})$ are $\frac{1}{2}$ - spin (isospin) operators defined by Pauli matrixes while $\vec{S}(\vec{T})$ operators correspond to $\frac{1}{2} \rightarrow \frac{3}{2}$ spin (isospin) transition. $m_{\pi}$ and $m_{\rho}$ are a pion and $\rho$-meson 


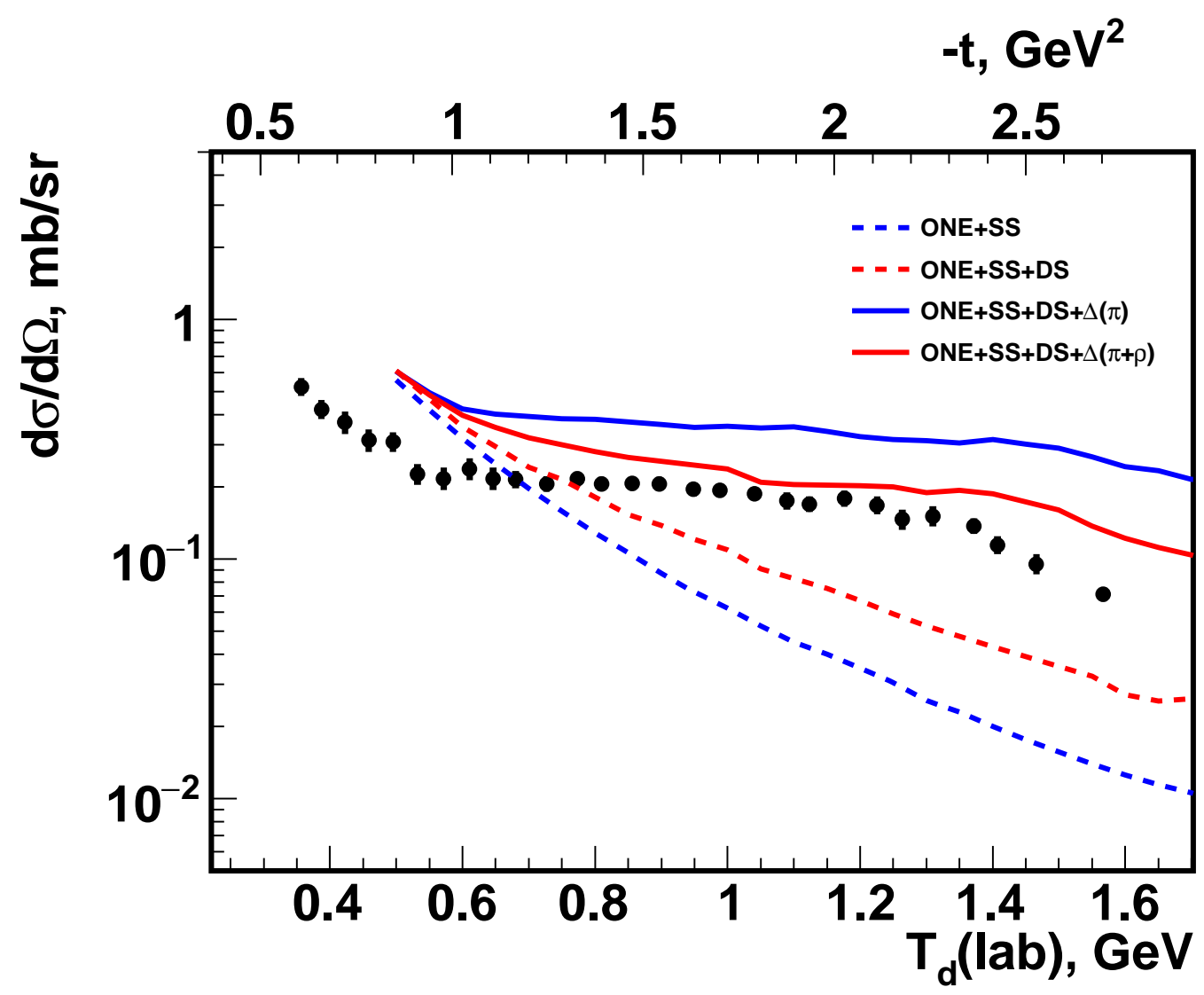

Figure 6: The energy dependence of the differential cross section at the scattering angle $\theta^{*}=180^{\circ}$. The data are from [24].

masses. The coupling constant $f_{\pi}$ is related with the $N N \pi$ vertex and $f_{\pi}^{*}$ corresponds to the $N \Delta \pi$ one. It concerns also $\rho-$ coupling constants.

$$
\begin{aligned}
& f_{\pi}=1.008 \quad f_{\pi}^{*}=2.156 \\
& f_{\rho}=7.8 \quad f_{\rho}^{*}=1.85 f_{\rho}
\end{aligned}
$$

The hadronic form factor was chosen in a pole form:

$$
F_{x}(t)=\left[\left(\Lambda_{x}^{2}-m_{x}^{2}\right) /\left(\Lambda_{x}^{2}-t\right)\right]^{n}
$$

In our calculation we use $\Lambda_{\pi}=0.8 \mathrm{GeV}, \Lambda_{\rho}=1.8 \mathrm{GeV}$. The exponent $n$ is equal to 1 for $\pi$-meson and 2 for $\rho$-meson.

Since two nucleon states in the $N N \rightarrow N \Delta$ vertexes are antisymmetrized, two permutation operators appear in Eq.(16). As consequence, the $\Delta$ - amplitude contains four terms: one direct, two exchange, and one double-exchange ones. The permutation operator $P_{i j}$ involves the permutation of all quantum numbers. Here, it is permutation over momentum, spin, and isospin indexes: $P_{i j}=P_{i j}(p) P_{i j}(\sigma) P_{i j}(\tau)$.

\section{Results}

We applied the method to describe angular dependences of the differential cross sections at the backward scattering angles $\theta^{*} \geq 140^{\circ}$ at four deuteron energies of $880,1000,1200$, and 


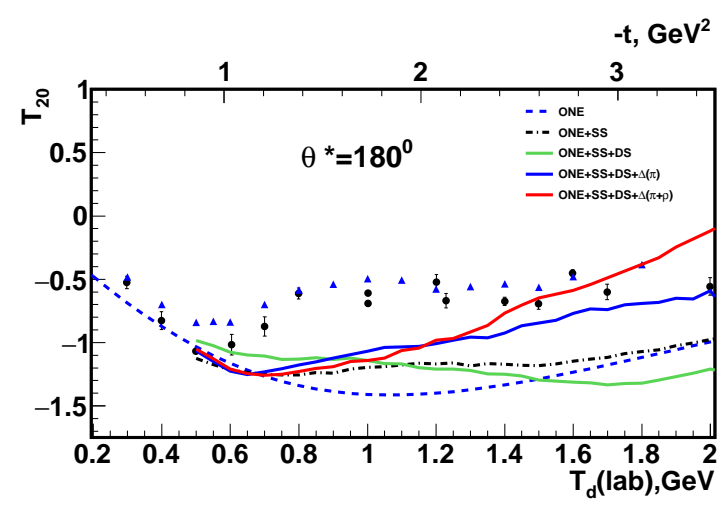

Figure 7: The energy dependence of the differential cross section at the scattering angle $\theta^{*}=180^{\circ}$. The data are taken from $\bullet-[25], \boldsymbol{\Delta}-[26]$.

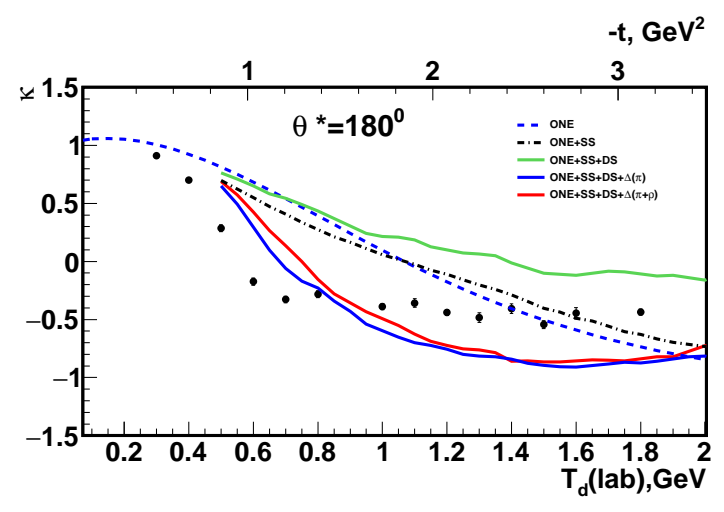

Figure 8: The energy dependence of the polarisation trnsfer at the scattering angle $\theta^{*}=180^{\circ}$. The data are taken from [26].

$1300 \mathrm{MeV}$. In Figs.2-5 the calculation results are presented for four cases. The dashed-dotted curves correspond to the results obtained taking into account only ONE and single-scattering contributions. The results obtained with the addition of the double-scattering term are represented by dashed curves. The solid curves correspond to the theory predictions taking into account also $\Delta$-isobar in the intermediate state with both $\pi$-meson (blue line) and $\pi$-and $\rho$-mesons (red line).

It is well known that the data on the differential cross sections show some enhancement at the backward angles. However the calculation results obtained without the $\Delta$-isobar lie below the data. Moreover the difference between the data and the results increases with the energy growing. When we include the $\Delta$-isobar into consideration we get rather good agreement between the data and theory in the case when $\pi$ - and $\rho$-mesons are taken into account. When we include only $\pi$-meson in the $\Delta$-isobar description we get overestimated values for the differential cross sections.

It is interesting to look at a manifestation of the various mechanisms at the critical scattering angle of $180^{\circ}$. An energy dependence of the differential cross section is presented in Fig.6. The data demonstrate a shoulder at the energies between about 500 and $1400 \mathrm{MeV}$ which is not described by the calculations without $\Delta$-isobar. The curves obtained taking into account only ONE+SS and ONE+SS+DS rapidly descend and pass below the data. Inclusion $\Delta$-isobar into consideration allows us to describe the shoulder and significantly improve an agreement between the data and theoretical predictions. As in the case of the angular distributions of the differential cross sections, we get overestimated values when we include only $\pi$ - meson in the $\Delta$-isobar definition.

Tensor analyzing power $T_{20}$ is presented in Fig. 6 as a function of the deuteron energy. The result of the simplest reaction mechanism ONE is close to the data at low energies up to about $500 \mathrm{MeV}$. But then the data go up while ONE curve descends up to minimum equal to $-\sqrt{2}$ at the deuteron energy of about $1 \mathrm{GeV}$. The addition of the single- and double- scattering terms allows to slightly rise the curves in the minimum but the data description remains unsatisfactory. The results obtained with $\Delta$-isobar are closer to the data except for the energy range between 700 and $1100 \mathrm{MeV}$ where the data show the rise.

The role of the $\Delta$-isobar is clearly manifested in the polarization transfer $x$, which is shown in Fig. 8 versus the deuteron energy. The data show the precipitous fall at the deuteron energy between 400 and $600 \mathrm{MeV}$ and then go on a plateau. The results obtained with the inclusion of $\Delta$-isobar reproduce the shape of the data while the results of the calculations performed without $\Delta$-isobar are far from the data. 


\section{Conclusion}

We considered dp- backward elastic scattering taking into account four contributions: onenucleon-exchange, single-scattering, double-scattering, and $\Delta$-excitation in an intermediate state. We showed a role of each reaction mechanism in the description of the angular dependence of the differential cross section. Inclusion of the $\Delta$-isobar term into consideration allowed to describe the enhancement of the differential cross section at $\theta^{*} \geq 140^{\circ}$ in the energy range between 880 and $1300 \mathrm{MeV}$.

The reaction mechanisms were also studied at the scattering angle $\theta^{*}=180^{\circ}$. It was obtained a quite good agreement between the experimental data and the theoretical predictions for the energy dependence of the differential cross section. Some progress was achieved in the description of the tensor analyzing power $T_{20}$ and polarisation transfer $\kappa$.

\section{Acknowledgements}

The author is grateful to Dr. V.P. Ladygin for fruitful discussions and interest in this problem. This work has been supported by the Russian Foundation for Basic Research under grant No 19-02-00079a.

\section{References}

[1] W.Glöckle et al., The tree-nucleon continuum:achievements, challenges and applications, Phys.Rep.274, 107 (1996),doi:10.1016/0370-1573(95)00085-2.

[2] K.Sekiguchi et al., Complite set of deuteron analyzing powers from $\vec{d} p$ elastic scattering at 190 MeV/nucleon, Phys.Rev. C96, 064001 (2017),doi:10.1103/PhysRevC.96.064001.

[3] V.Franco, Small-angle high-energy scattering by deuterons, Phys.Rev.Lett. 16, 944-947 (1966), doi:10.1103/PhysRevLett.16.944

[4] V.Franco, E.Coleman, Double scattering in high-energy elastic collisions with deuterons, Phys.Rev.Lett. 17, 827-830 (1966)doi:10.1103/PhysRevLett.17.827

[5] A.K. Kerman, L.S. Kisslinger, High-energy backward elastic proton-deuteron scattering and baryon resonances, Phys. Rev. 180, 1483 (1969), doi:10.1103/PhysRev.180.1483 .

[6] L.A. Kondratyuk, F.M. Lev, P d Backward Scattering in Resonance Region, Sov. J. Nucl. Phys. 26, 153 (1977).

[7] L.A. Kondratyuk, F.M. Lev, L.V. Shevchenko, On pd backward elastic scattering in the $\Delta$ resonance region and the one-pion exchange form factor, Sov. J. Nucl. Phys. 29, 558 (1979).

[8] L.A. Kondratyuk, F.M. Lev, L.V. Shevchenko, Large angle nucleon deuteron scattering and three baryon resonances, Yad. Fiz. 33, 1208 (1981).

[9] A. Deltuva, K. Chmielewski, P.U. Sauer, Nucleon deuteron scattering with delta -isobar excitation: Chebyshev expansion of two baryon transition matrix, Phys. Rev. C 67, 034001 (2003), doi:10.1103/PhysRevC.67.034001 . 
[10] A. Deltuva, R. Machleidt, P.U. Sauer, Realistic two baryon potential coupling two nucleon and nucleon delta isobar states: Fit and applications to three nucleon system, Phys. Rev. C 68, 024005 (2003), doi:10.1103/PhysRevC.68.024005 .

[11] N.B. Ladygina, Deuteron-proton elastic scattering at intermediate energies, Phys. Atom. Nucl. 71, 2039 (2008), doi:10.1134/S1063778808120053.

[12] N.B. Ladygina, Differential Cross Section of DP-Elastic Scattering at Intermediate Energies, Eur. Phys. J. A42, 91 (2009), doi:10.1140/epja/i2009-10852-0.

[13] N.B. Ladygina, Delta excitation in deuteron-proton elastic scattering, Eur. Phys. J. A52, 199 (2016), doi:10.1140/epja/i2016-16199-5.

[14] R.Machleidt, K.Holinde, Ch.Elster,The Bonn meson exchange model for the nucleon nucleon interaction Phys.Rep. 149, 1 (1987), doi:10.1016/S0370-1573(87)80002-9 .

[15] R.Machleidt, The High precision, charge dependent Bonn nucleon-nucleon potential (CDBonn), Phys. Rev. C63, 024001 (2001), doi:10.1103/PhysRevC.63.024001.

[16] M. Lacombe et al., Parametrization of the deuteron wave function of the Paris n-n potential, Phys.Lett. B101, 139 (1981), doi: 10.1016/0370-2693(81)90659-6.

[17] W.G.Love, M.A.Franey (1981), Effective nucleon nucleon interaction for scattering at intermediate-energies, Phys. Rev. C24, 1073, doi: 10.1103/PhysRevC.24.1073;Erratum: Phys.Rev.C27, 438 (1983), doi: 10.1103/PhysRevC.27.438 .

[18] N.B.Ladygina (2008), e-preprint nucl-th/0805.3021

[19] http://gwdac.phys.gwu.edu

[20] J.C.Alder et al,Elastic pd-scattering at 316, 364, 470, and $590 \mathrm{MeV}$ in the backward hemisphere, Phys.Rev.C6, 2010 (1972), doi: 10.1103/PhysRevC.6.2010.

[21] N.E.Booth et al.,Proton-deuteron elastic scattering at $1.0 \mathrm{GeV} / \mathrm{c}$, Phys.Rev.D4, 1261 (1971), doi: 10.1103/PhysRevD.4.1261 .

[22] J.S.Vincent, Large angle p-d scattering at 580 mev, Phys.Rev.Lett.24, p.236 (1970), doi: 10.1103/PhysRevLett.24.236.

[23] E.T.Boschitz et al.,Elastic scattering of $600-\mathrm{MeV}$ protons from $\mathrm{H}, \mathrm{D},{ }^{3} \mathrm{He}$, and ${ }^{4} \mathrm{He}$, Phys.Rev.C6, 457 (1972), doi: 10.1103/PhysRevC.6.457.

[24] B.E.Bonner et al., $n$ d Scattering at $180^{\circ}$ for neutron energies from $200-\mathrm{MeV}$ to $800-\mathrm{MeV}$, Phys.Rev.Lett.39, p.1253 (1977), doi:10.1103/PhysRevLett.39.1253 .

[25] J.Arvieux et al., Elastic scattering of polarized deuterons by protons at intermediateenergies, Nucl.Phys.A431, p.613 (1984), doi:10.1016/0375-9474(84)90272-0.

[26] V.Punjabi et al., Measurement of polarization transfer $\kappa_{0}$ and tensor analyzing power $T_{20}$ in the backward elastic d p scattering, Phys.Let.B350, p.178 (1995), doi: 10.1016/0370-2693(95)00344-K. 